\title{
Deconvolution of in vivo ultrasound images
}

\author{
Jensen, Jørgen Arendt
}

Published in:

Proceedings of Ultrasonics Symposium

Link to article, DOI:

10.1109/ULTSYM.1990.171635

Publication date:

1990

Document Version

Publisher's PDF, also known as Version of record

Link back to DTU Orbit

Citation (APA):

Jensen, J. A. (1990). Deconvolution of in vivo ultrasound images. In Proceedings of Ultrasonics Symposium (Vol. Volume 3, pp. 1581-1587). IEEE. https://doi.org/10.1109/ULTSYM.1990.171635

\section{General rights}

Copyright and moral rights for the publications made accessible in the public portal are retained by the authors and/or other copyright owners and it is a condition of accessing publications that users recognise and abide by the legal requirements associated with these rights.

- Users may download and print one copy of any publication from the public portal for the purpose of private study or research.

- You may not further distribute the material or use it for any profit-making activity or commercial gain

- You may freely distribute the URL identifying the publication in the public portal

If you believe that this document breaches copyright please contact us providing details, and we will remove access to the work immediately and investigate your claim. 


\title{
DECONVOLUTION OF IN VIVO ULTRASOUND IMAGES
}

\author{
Jørgen Arendt Jensen \\ Electronics Institute, build. 349 \\ Technical University of Denmark, \\ DK-2800 Lyngby, Denmark
}

\begin{abstract}
The appearance of an ultrasound image is intimately linked to the nature of the emitted pressure field and its distortion down through the tissue. The pulse field is temporally and spatially varying due to focusing and the dispersive attenuation.

The influence of the pulse and attenuation should be removed from the picture in order to display a more consistent and uniform image.

This paper describes an algorithm to remove the influence of the attenuated pulse on the image. The algorithm takes into account the varying pulse, noise in the acquired signal and the changing reflectivity in the tissue. Both one- and two-dimensional processing can be implemented. The algorithm relies on prior knowledge of the pulse, and of the covariance of the noise and the reflections. Algorithms to estimate these are given.

Examples of use are presented. One-dimensionally processed images of the kidney and a foetus are shown.
\end{abstract}

\section{Introduction}

Ultrasound is routinely used in hospitals for the investigation of nearly all soft tissue structures of the human body. Advanced transducers (phased and linear array) are used in order to acquire sharp images. Improved resolution is steadily sought by increasing the center frequency and bandwidth of the emitted pulse. Increasing the center frequency, however, limits the depth of penetration due to the dispersive attenuation of the pulse in the tissue. This limits the resolution attainable, and to further increase resolution, or depth of penetration for the same resolution, we must turn to digital processing, i.e. deconvolution.

Several authors have investigated techniques for enhancing the resolution of ultrasound images. Fatemi and Kak [1] derived an expression for the received pressure field and suggested use of a Wiener filter [2]. This has also been suggested in [3], [4]. Slightly different, but conceptually similar methods have been suggested in $[5],[6]$,
[7] [8]. These estimators use, however, fixed parameters for the emitted pulse and can not take into account the spatially varying source wavelet and the dispersive attenuation, which are important factors for in vivo images. We therefore turn to other, more advanced estimators.

In this paper we will devise an algorithm, based on physical reasoning, that can take into account variations in pulse shape, signal-to-noise ratio and reflection strength. It is based on Mendel's fixed-interval deconvolution algorithm [9], [10], which calculates an optimal, minimum variance estimate of the reflections. As this estimator can handle varying parameters optimally, the original problem is transformed into finding parameters to describe the pulse and covariances for the noise and the reflections. These parameter estimators should take into account temporal and spatial variations, and section 4 and 5 will explain how to do this.

Examples of the use of the combined algorithm are shown both for data acquired from a tissue mimicking phantom and for clinical in vivo data. These demonstrate the algorithm's capability to increase the resolution and to handle variations in reflection strength. The effect of non-linearities emanating from the data acquisition and ways of avoiding them are discussed.

\section{The general algorithm}

It can be shown that the received pressure field can be calculated by [11]:

$$
p_{r}\left(\vec{r}_{2}, t\right)=v_{p e}(t) \underset{t}{\star} f_{m}\left(\vec{r}_{1}\right) \underset{r}{\star} h_{p e}\left(\vec{r}_{1}, \vec{r}_{2}, t\right)
$$

where $\stackrel{\star}{t}$ denotes a temporal and $\stackrel{\star}{r}$ a spatial convolution. $v_{p e}$ is the pulse-echo wavelet, that accounts for the transducer excitation and the electro-mechanical impulse response during emission and reception of the pulse. $f_{m}$ accounts for inhomogeneities in the tissue due to density and propagation velocity perturbations, which gives rise to the scattered signal. $h_{p e}$ is the modified pulse-echo spatial impulse response, that relates the 
transducer geometry to the spatial extent of the scattered field. Explicitly written out it is:

$$
h_{p e}\left(\vec{r}_{1}, \vec{r}_{2}, t\right)=\frac{1}{c_{0}^{2}} \frac{\partial h\left(\vec{r}_{1}, \vec{r}_{2}, t\right)}{\partial t} \star \frac{\partial h\left(\vec{r}_{1}, \vec{r}_{2}, t\right)}{\partial t}
$$

$\vec{r}_{1}$ denotes the position of the scatterer and $\vec{r}_{2}$ the position of the transducer. $h$ is the spatial impulse response for the transducer geometry as calculated by the Tupholme-Stepanishen method [12],[13],[14]. Equation (1) was derived under the assumption of weak scattering by density and propagation velocity perturbations in the tissure, and absorption and multiple scattering were neglected. The dispersive attenuation can be lumped into $v_{p e}$, that then is substituted by a pulse, $V_{p e a}\left(t, \vec{r}_{1}, \vec{r}_{2}\right)$ that depends on distance.

Expression (1) consists of three distinct terms. The signal of interest, and the one that should be displayed in medical ultrasound, is $f_{m}\left(\vec{r}_{1}\right)$. We, however, measure a time and spatially smoothed version of this, which obscures the finer details in the image. The smoothing consists of a convolution in time with a wavelet $v_{\text {pea }}\left(t, \vec{r}_{1}, \vec{r}_{2}\right)$, and a spatial convolution with a spatially varying $h_{p e}\left(\vec{r}_{1}, \vec{r}_{2}, t\right)$.

It is necessary to employ digital signal processing to make a better estimation of $f_{m}$. Thus, (1) is reformulated in a discrete form.

$$
\begin{gathered}
y\left(\vec{r}_{d_{2}}, k\right)=v_{p e a}\left(k, \vec{r}_{d_{1}}, \vec{r}_{d_{2}}\right) \underset{k}{\star} f_{m}\left(\vec{r}_{d_{1}}\right) \\
\star{ }_{r_{d}} h_{p e}\left(\vec{r}_{d_{1}}, \vec{r}_{d_{2}}, k\right)+n\left(\vec{r}_{d_{2}}, k\right)
\end{gathered}
$$

$k$ is the discrete time variable, and $\vec{r}_{d}$ is a discrete vector. A noise term $n\left(\vec{r}_{d_{2}}, t\right)$ has been added to explain the inevitable noise in the measured signal. The term accounts for both electrical noise from amplifiers and for physical effects not explained by the convolution model.

We now seck a procedure to obtain an estimate of $f_{m}\left(\vec{r}_{d_{1}}\right)$. This can formally be stated as [15]:

$$
\begin{gathered}
\hat{f}\left(\vec{r}_{d_{1}}\right)=\mathcal{F}\left[y\left(\vec{r}_{d_{2}}, k\right), v_{p e a}\left(k, \vec{r}_{d_{1}}, \vec{r}_{d_{2}}\right),\right. \\
\left.h_{p e}\left(\vec{r}_{d_{1}}, \vec{r}_{d_{2}}, k\right), n\left(\vec{r}_{d_{2}}, k\right)\right]
\end{gathered}
$$

The estimate $f_{m}\left(\vec{r}_{d_{1}}\right)$ is a function of the measured signal $y\left(\vec{r}_{d_{2}}, k\right)$ and some knowledge, measured or estimated, about $v_{p e a}, h_{p e}$, and $n$.

The function $\mathcal{F}$ can be a linear or non-linear mapping from $y, v_{p e a}, h_{p e}$, and $n$ to $\hat{f}_{m}$. It can be evaluated directly or through iterative techniques. One of the advantages of ultrasound $\mathrm{B}$-mode systems is real-time image formation. This precludes an iterative search scheme, as in a real-time system only a fixed amount of calculations can be carried out per sample. Rather the estimate $\hat{f}_{m}\left(\vec{r}_{d_{1}}\right)$ is based on some estimate of the internal state of the system so that

$$
\begin{aligned}
\hat{S}\left(\vec{r}_{d_{2}}, k\right)= & \mathcal{H}\left[y\left(\vec{r}_{d_{2}}, k\right), v_{p e a}\left(k, \vec{r}_{d_{1}}, \vec{r}_{d_{2}}\right),\right. \\
& \left.h_{p e}\left(\vec{r}_{d_{1}}, \vec{r}_{d_{2}}, k\right), n\left(\vec{r}_{d_{2}}, k\right)\right] \\
\hat{f}_{m}\left(\vec{r}_{d_{1}}\right)= & \mathcal{G}\left[\hat{S}\left(\vec{r}_{d_{2}}, k\right)\right]
\end{aligned}
$$

where $\mathcal{H}$ linearly relates $y, v_{p e a}, h_{p e}$, and $n$ to $\hat{S}$, and $\mathcal{G}$ linearly relates $\hat{S}$ to $\hat{f}_{m} . S$ is a vector of fixed dimension that represents the state of the system. $\mathcal{H}$ and $\mathcal{G}$ can be evaluated with a fixed and a priori known number of calculations. This allows for a time-varying model, where each sample can be treated in a fixed, predetermined interval of time [15]:

\section{The deconvolution algorithm}

Eq. (5) explains in general terms how to remove $v_{p e a}$ and $h_{p e}$ from $y$ to obtain $f_{m}$. We will now detail how to perform this deconvolution, when the parameters are known.

The algorithm used is Mendel's fixed-interval deconvolution estimator. It consists of two parts: first a Kalman filtering is performed on the data time-recursively, and then a subsequent estimation step performed backwards time-recursively. The result is a minimum variance, fixed-interval estimate of the reflection signal. The algorithm can be used on one A-line or on a number of A-lines at a time, and thus perform two-dimensional deconvolution.

For easy reference the basic equations are now given. The Kalman filter is based on the following time-varying state-space model:

$$
\begin{aligned}
x(k+1) & =\Phi(k+1, k) x(k)+\Gamma(k+1, k) w(k+1) \\
z(k) & =H^{T}(k) x(k)+n(k)
\end{aligned}
$$

$z(k)$ is the measured signal, $w(k+1)$ is the "tissue" reflection signal, $x(k)$ the state vector, and $n(k)$ the measurement noise. $\Phi$ is the state transition matrix, $\Gamma$ the input distribution matrix, and $H$ the output matrix. Note the direct coupling between the input $w(k)$ and $z(k)$. The equations describe a time-varying model, for which a new set of coefficients in the state matrices and vectors can be used for each time instance. It is assumed that the $w(k+1)$ and $n(k)$ are zero mean, Gaussian signals with a covariance of $Q(k+1)$ and $R_{n}(k)$.

The Kalman filter is, stated in the predictor-corrector form:

\section{Predictor equations}

$\hat{x}(k+1 \mid k)=\Phi(k+1, k) \hat{x}(k \mid k)$

\section{2 - 1990 ULTRASONICS SYMPOSIUM}




$$
\begin{aligned}
P(k+1 \mid k)= & \Phi(k+1, k) P(k \mid k) \Phi^{T}(k+1, k)+ \\
& \Gamma(k+1, k) Q(k+1) \Gamma^{T}(k+1, k)
\end{aligned}
$$

\section{Corrector equations}

$$
\begin{aligned}
K(k+1)= & P(k+1 \mid k) H(k+1) \cdot \\
& {\left[H^{T}(k+1) P(k+1 \mid k) H(k+1)\right.} \\
& \left.+R_{n}(k+1)\right]^{-1} \\
z(k+1 \mid k)= & z(k+1)-H^{T}(k+1) \hat{x}(k+1 \mid k) \\
x(k+1 \mid k+1)= & \hat{x}(k+1 \mid k)+K(k+1) \tilde{z}(k+1 \mid k) \\
P(k+1 \mid k+1)= & {\left[I-K(k+1) H^{T}(k+1)\right] . } \\
& P(k+1 \mid k)
\end{aligned}
$$

$P(k+1 \mid k)$ is the a-priori state covariance, and $P(k+1 \mid$ $k+1)$ the a-posteriori covariance. $K(k)$ is the Kalman gain.

The backwards time-recursive estimation step can be expressed as:

$$
\begin{aligned}
\hat{w}(k+1 \mid N)= & Q(k+1) \Gamma^{T}(k+1, k) r(k+1 \mid N) \\
r(k \mid N)= & H(k)\left[H^{T}(k) P(k \mid k-1) H(k)\right. \\
& \left.+R_{n}(k)\right]^{-1} \tilde{z}(k \mid k-1)+(\Phi(k+1, k) . \\
& {\left.\left[I-K(k) H^{T}(k)\right]\right)^{T} r(k+1 \mid N) }
\end{aligned}
$$

$w(k+1 \mid N)$ denotes the fixed-interval estimate of $w(k+1) \cdot r(k+1 \mid N)$ is the residual state vector [9].

The algorithm can, based on all samples in one or more A-lines, estimate the reflections. The pulse parameters and the covariances can vary from sample to sample and the pulse can be non-minimum phase [9].

\section{Fixed-interval pulse estimator}

In (5) knowledge of $v_{p e a}$ and $h_{p e}$ is needed. $v_{p e a}$ must be stimated because, in general, dispersive attenuation is unknown. $h_{\text {pe }}$ can be calculated from a priori knowledge of the transducer geometry.

A representative model for $v_{p e a}$ is the ARMA (AutoRegressive Moving Average) model given by:

$$
\left(1+a_{1} q^{-1} \cdots+a_{n_{a}} q^{-n_{a}}\right) y(k)=\left(1+c_{1} q^{-1} \cdots+c_{n_{c}} q^{-n_{c}}\right) e(k)
$$

where $y(k)$ is the measured signal, $e(k)$ the reflection signal, $q^{-1}$ the unit backward shift operator, and $a_{1}, \ldots, a_{n_{\alpha}}$ and $c_{1}, \ldots, c_{n_{c}}$ are the ARMA coefficients.

The parameters to be estimated, and the data values, are, for notational convenience, organised in a parameter vector, $\theta$, and a regression vector, $\varphi$.

$$
\theta^{T}=\left[a_{1}, a_{2}, \ldots, a_{n_{a}}, c_{1}, c_{2}, \ldots, c_{n_{c}}\right]
$$

$\varphi^{T}(k)=\left[-y(k-1), \ldots,-y\left(k-n_{a}\right), e(k-1), \ldots, e\left(k-n_{c}\right)\right]$

Then the output is written as:

$$
y(k)=\varphi^{T}(k) \theta+e(k)
$$

8) and the prediction and prediction error as:

$$
\begin{aligned}
\hat{y}(k \mid \theta) & =\varphi^{T}(k) \theta \\
\hat{e}(k, \theta) & =y(k)-\hat{y}(k \mid \theta)
\end{aligned}
$$

The parameters are found by minimizing the magnitude of the prediction error. This is formulated as:

$$
\begin{gathered}
V_{N}(\theta, \mathcal{Z}(N))=\frac{1}{N} \sum_{k=1}^{N} \hat{e}(k, \theta) \hat{e}(k, \theta) \\
\hat{\theta}_{N}=\operatorname{Arg} \min V_{N}(\theta, \mathcal{Z}(N))
\end{gathered}
$$

stating that $V_{N}$ is minimized with respect to $\theta$ under the constraint that the parameters belong to the set of stable models $D_{\mathcal{M}} . \mathcal{Z}(N)$ denotes the set of measure9) ments $\{y(1), y(2), \ldots, y(N)\}$. This parameter estimation method is called the prediction error method (PEM) [15], [16].

Using an iterative search algorithm employing the Gauss-Newton search gradient, we arrive at the following equations [16]:

START:

$$
\begin{aligned}
\psi(k, \theta) & =[1-C(q)] \varphi(k) \\
V_{N}^{\prime(i)}(\theta, \mathcal{Z}(N)) & =-\frac{1}{N} \sum_{k=1}^{N} \psi^{T}(k, \theta) \hat{e}^{(i)}(k, \theta) \\
V_{N}^{\prime \prime(i)}(\theta, \mathcal{Z}(N)) & =\frac{1}{N} \sum_{k=1}^{N} \psi^{T}(k, \theta) \psi(k, \theta) \\
\alpha & =1
\end{aligned}
$$

ITERATE:

$$
\hat{\theta}_{N}^{(i+1)}=\hat{\theta}_{N}^{(i)}+\alpha\left[V_{N}^{\prime \prime(i)}(\theta, \mathcal{Z}(N))\right]^{-1} V_{N}^{\prime(i)}(\theta, \mathcal{Z}(N))
$$

Mirror zeros and poles into the unit circle.

$$
\begin{aligned}
& \hat{e}^{(i+1)}(k, \theta)=y(k)-\varphi^{T}(k) \theta_{N}^{(i+1)} \\
& V_{N}^{(i+1)}(\theta, \mathcal{Z}(N))=\frac{1}{N} \sum_{k=1}^{N} \hat{e}^{(i+1)}(k, \theta) \hat{e}^{(i+1)}(k, \theta) \\
& \text { If } V_{N}^{(i+1)}<V_{N}^{(i)} \text { then go to START. } \\
& \text { else } \alpha=\alpha / 2 \text { and go to ITERATE with } \\
& \text { the new } \alpha \text { until tried ten times. If } \\
& \left\|\left[V_{N}^{\prime \prime(i)}(\theta, \mathcal{Z}(N))\right]^{-1} V_{N}^{\prime(i)}(\theta, \mathcal{Z}(N))\right\|<0.01 \text { or } \\
& i=10 \text { then stop }
\end{aligned}
$$


$i$ is the iteration step, and $\|\bullet\|$ denotes the norm of the vector.

Some initial parameter and prediction error estimates must be supplied. They are calculated by the leastsquares method.

1. Fit an AR-model of order $n_{a}+n_{c}$ to the data

$$
\begin{aligned}
& \text { Model: } A_{1}(q) y(k)=e(k) \\
& \hat{\theta}_{\mathrm{LS} 1}=\left[\frac{1}{N} \sum_{k=1}^{N} \varphi_{1}(k) \varphi_{1}^{T}(k)\right]^{-1} \frac{1}{N} \sum_{k=1}^{N} \varphi_{1}(k) y(k)
\end{aligned}
$$

2. Calculate the initial estimate of the prediction error

$$
\hat{e}\left(k, \theta_{\mathrm{LS} 1}\right)=y(k)-\varphi_{1}^{T}(k) \hat{\theta}_{\mathrm{LS} 1}
$$

3. Make a least squares fit to an ARX-model of order $n_{a}$ and $n_{c}$, where the external input is $\hat{e}\left(k, \theta_{\mathrm{LS} 1}\right)$

Model: $A(q) y(k)=C(q) e(k)$

This gives the initial parameter estimate for $\theta$ and the prediction error.

This estimator gives one set of parameters for a segment of an A-line. It can be reformulated to give one set for each sample [16] tracking the slow variation in the pulse. We, however, prefer to divide the A-line into overlapping segments, and then use parameters from the previous segment to initialize the next segment. This has given more consistent results than using the recursive prediction error method.

\section{Covariance estimation}

The deconvolution algorithm uses the covariance of the noise and of the reflections. The noise is due to the ana$\log$ amplifiers, the time gain amplifier and quantization of the signal. It can be modeled as a fixed noise source added to the noise from the TGC amplifier that varies with its gain factor. So the noise covariance can be found from previous measurement of the noise sources and the gain factor actually used for the acquired data,

When the signal-to-noise ratio is good we can approximate the measured signal by the ARMA model in (10). The reflections can then be approximately obtained by performing a predictive deconvolution by the ARMA filter and then finding the covariance. To reduce the variance of the covariance estimate several adjacent lines are used, and each line is weighted by a Hamming window.

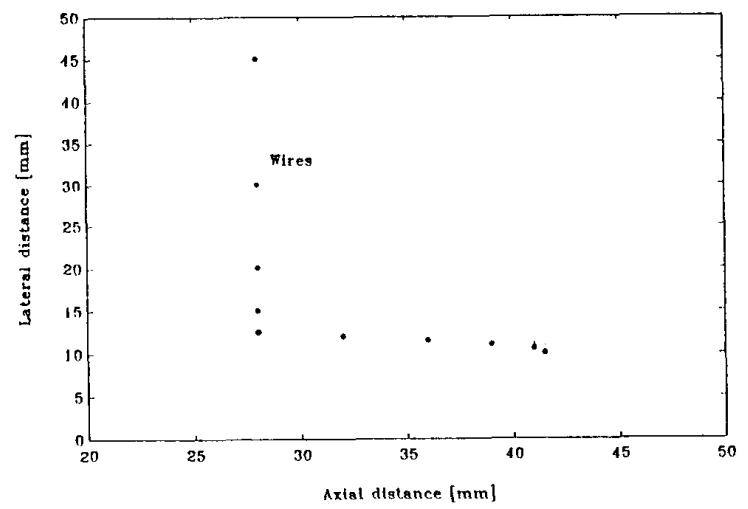

Figure 1: Position of wires.

\section{Phantom examples}

We will now show results from deconvolving signals obtained from a tissue phantom (Nuclear Associates). As we have not been able to create an invertible model for the pulse-echo spatial impulse response, only a 1D deconvolution will be performed with a fixed, estimated wavelet. As the data segment only covers a relatively small depth, a fixed value of $Q_{v_{r}}$ and $R_{v_{n}}$ will be used. The values are determined by the method described in the previous section.

The area scanned contained wires as shown in Fig. 1, embedded in a substrate which generates a speckle signal.

The transducer used was the Brüel \& Kjær 8526. The focal point is at $5 \mathrm{~cm}$ and the nominal frequency is 3.5 $\mathrm{MHz}$. The sampling frequency was $25 \mathrm{MHz}$.

The $\operatorname{ARMA}(6,5)$ wavelet used by the deconvolution algorithm was estimated from one of the acquired lines which contained only a speckle signal. The wavelet is shown in Fig. 2.

Using the approach in section 5 , the ratio $Q_{v_{r}} / R_{v_{n}}$ was estimated to be 9.2 , and this was used in the deconvolution. The signal-to-noise ratio was estimated to 43.

Fig.3 shows the logarithmic envelope of the signals, found by Hilbert transformation. The picture at the top is the normally processed picture, as displayed on a modern scanner.

Fig. 3 shows that the speckle pattern is suppressed and the wires are more distinct than in the traditional picture. This makes it easier to distinguish between speckular scatterers and reflections as the contrast in the picture is enhanced. 


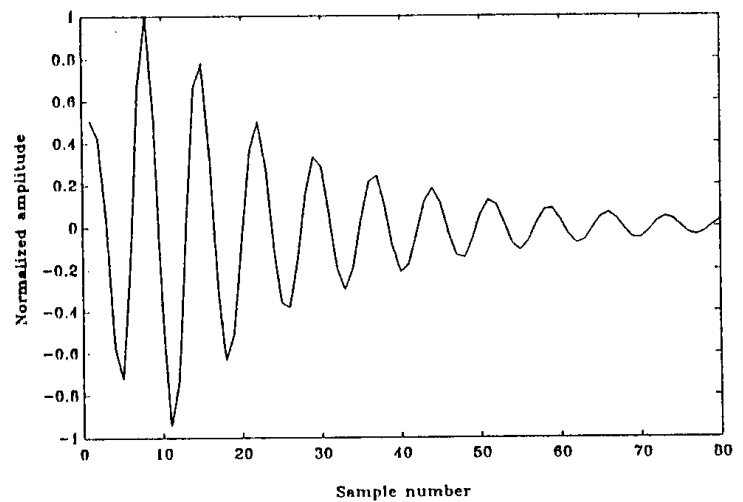

Figure 2: Estimated wavelet for the 8526 Brüel \& Kjær transducer.

\section{$7 \quad$ In vivo examples}

We now present two examples with clinical in vivo data. The data were acquired by our own prototype sampling system that uses a 12 bits, $20 \mathrm{MHz}$ converter. The measurements were performed at Herlev University Hospital in Denmark with a Brüel \& Kjær 1846 scanner with a $85263 \mathrm{MHz}$ sector scan transducer.

The first image shown in Fig. 4 is the lower part of the right kidney in a longitudinal scan.

Although the change is not as pronounced as for the phantom data, we do see an improvement. The speckle pattern is finer, and it seems easier to identify the exact boundary of the kidney. The internal structure of the kidney also seems more clearly identified from the speckle noise.

The second example shown in Fig. 5 is a 13 weeks foetus . The head, limbs, and spinal cord can clearly be identified. The deconvolved picture shows the same changes, a slightly finer speckle pattern with, apparently easier identification of the extent of the placenta.

One feature, however, stands out in the deconvolved image; the diamond shaped, white area behind the head. This is due to overload of the input amplifier in the scanner due to a very large reflection. The algorithm relies on a linearity assumption, which is violated here. Thus, the measurement process should ensure that no non-linearities occur. This should not be done by adjusting the TGC for the whole picture, rather an adaptive TGC for each individual line should be used. Thereby a relatively large signal-to-noise ratio can be obtained for the whole image.
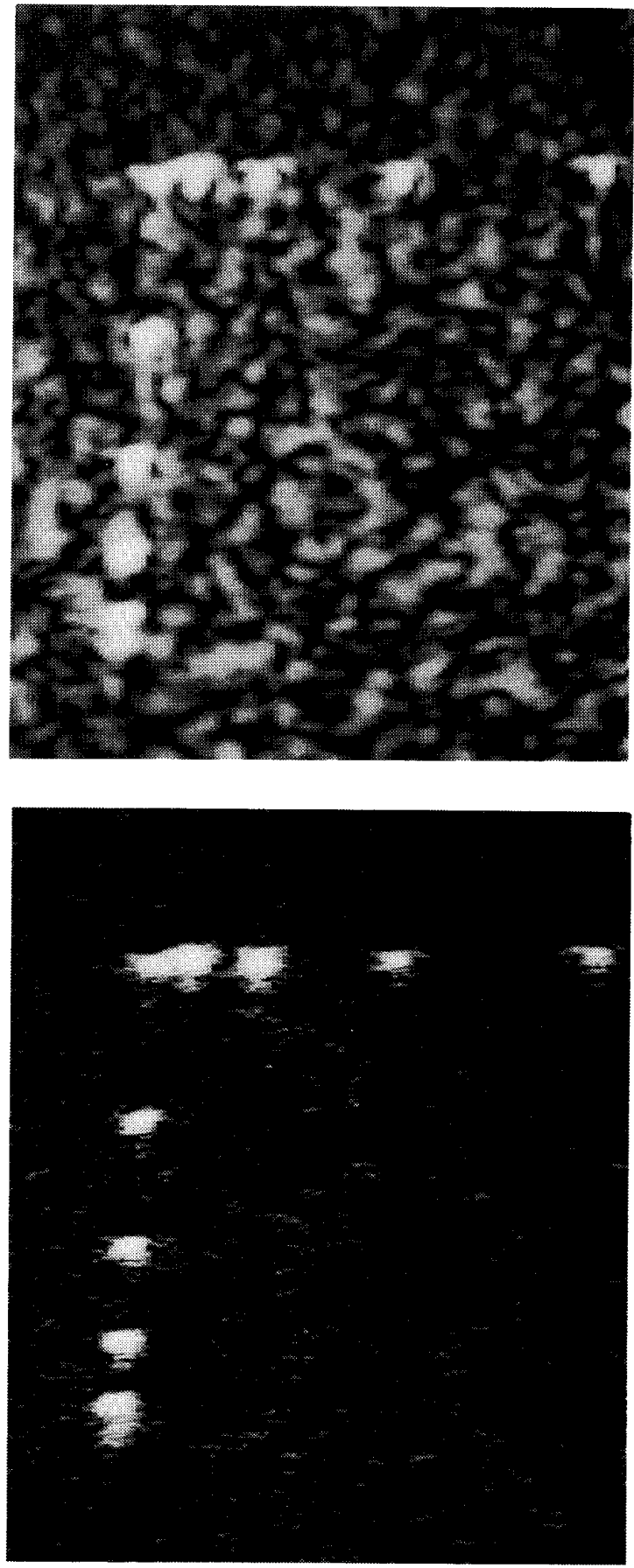

Figure 3: Normal and deconvolved response for image measured by the Brüel \& Kjær 8526 transducer. Axial distance $30 \mathrm{~mm}$ and lateral distance $50 \mathrm{~mm}$. Linear scan. 

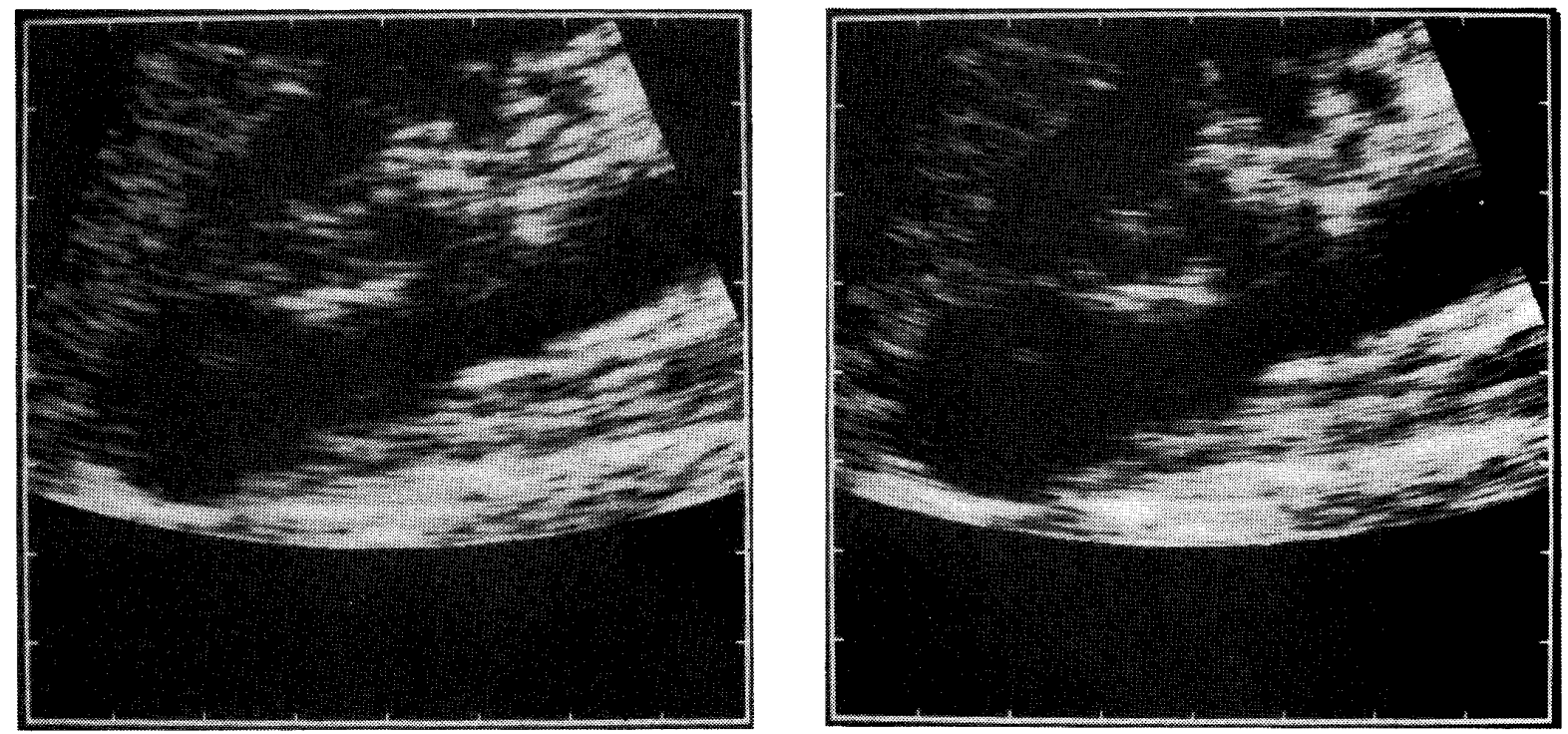

Figure 4: Normal (left) and deconvolved (right) image of longitudinal scan of right kidney. $1 \mathrm{~cm}$ between markers.
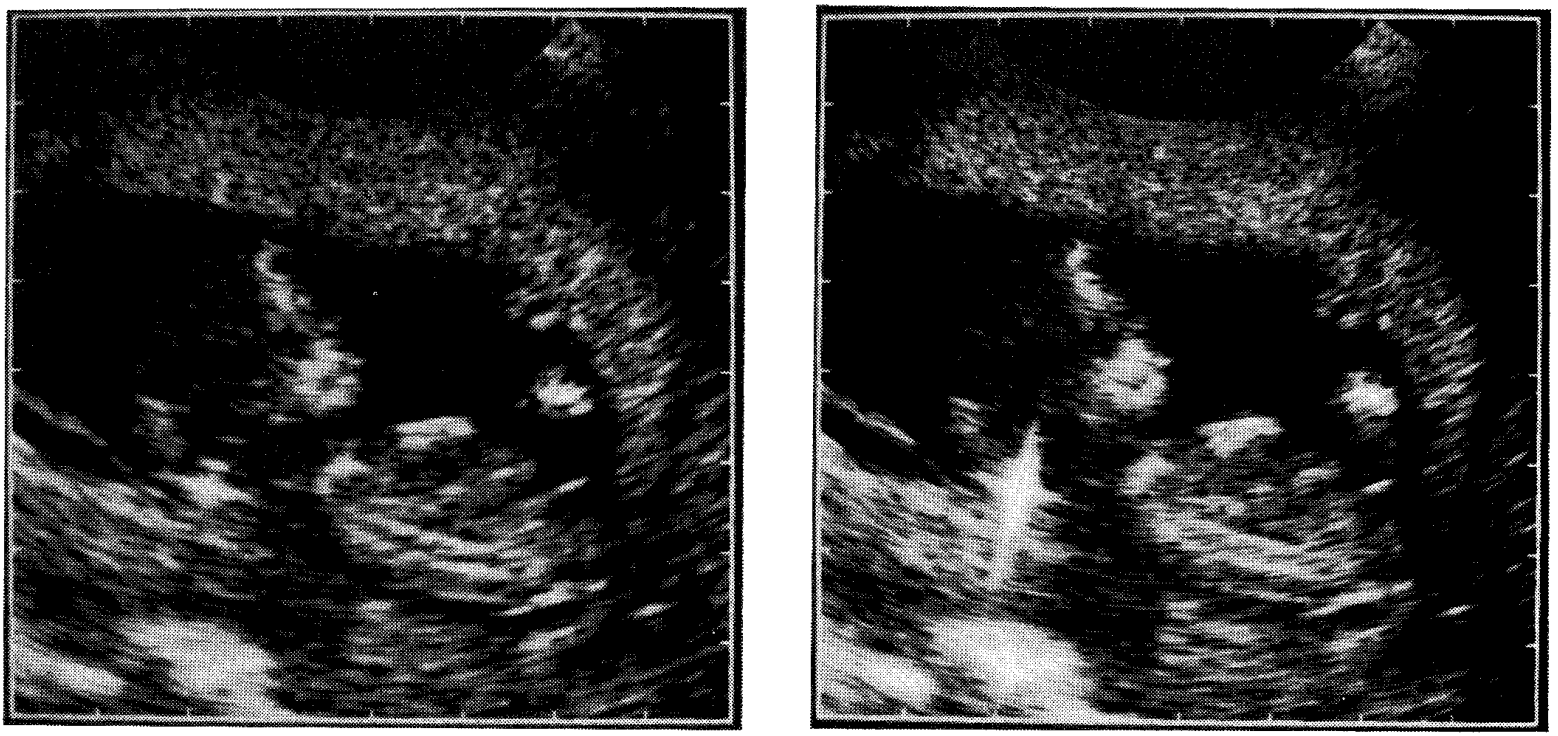

Figure 5: Normal (left) and deconvolved (right) image of 13 weeks old foetus. $1 \mathrm{~cm}$ between markers. 


\section{Conclusion}

An optimal, fixed-interval, minimum variance algorithm for increasing the resolution of medical ultrasound pictures was given. It can take into account the spatial and temporal change in the emitted and received pressure field and changes in the noise and reflection covariance.

The algorithm uses knowledge of the interrogating pulse and of the covariances, and estimators for the onedimensional pulse and for the covariances were given.

The algorithm can handle two-dimensional deconvolution, but currently we have not been able to obtain a stable and invertible 2D model for the pressure field.

Examples of $1 \mathrm{D}$ deconvoluted pictures of phantom dalia and in vivo data were given. They showed, especially for the phantom data, an increased contrast and resolution. Problems were encountered with in vivo images due to non-linearities in the acquired signals. It is suggested that this can be solved by using adaptive time gain compensation selected for each line in the image.

\section{Acknowledgement}

The research was partly funded by the Danish Technical Research Council, grant 16-4218.E, Brüel \& Kjær A/S, Novo's Foundation, H.C. Ørsteds Foundation and Trane's Foundation.

M.Sc. Jan Mathorne constructed the sampling system and M.Sc. Torben Gravesen and Dr. Bjarne Stage wrote the programs for the data acquisition.

Dr. Sidney Leeman, King's College London is thanked for proofreading the manuscript.

\section{References}

[1] M.Fatemi and A.C.Kak: Ultrasonic B-scan imaging: Theory of image formation and a technique for restoration, Ultrasonic Imaging 2, pp. 1-47, 1980.

[2] N.Wiener: Extrapolation, interpolation and smoothing of stationary time series, with engineering applications, Wiley \& Sons, Inc., New York, 1949.

[3] C.N.Liu, M.Fatemi and R.C.Waag: Digital processing for improvement of ultrasonic abdominal images, IEEE Trans. Med. Imag., vol. MI-2, No. 2, pp. 66-75, June 1983.

[4] D.E.Robinson and M.Wing: Lateral deconvolution of ultrasonic beams, Ultrasonic Imaging 6, pp. 1-12, 1984.

[5] E.E.Hundt and E.A.Trautenberg: Digital processing of ultrasonic data by deconvolution, IEEE Trans.
Son. Ultra., vol.SU-27, No.5, pp. 249-252, September 1980 .

[6] A.Herment, G.Demoment and M.Vaysse: Algorithm for on line deconvolution of echographic signals, Acoustical Imaging, vol.10, pp. 325-345, 1980.

[7] W.Vollmann: Resolution enhancement of ultrasonic $B$-scan images by deconvolution, IEEE Trans. Son. Ultra., vol. SU-29, No. 2, pp. 78-83, March 1982.

[8] G.Demoment, R.Reynaud and A.Herment: Range resolution improvement by a fast deconvolution. method, Ultrasonic Imaging 6, pp. 435-451, 1984.

[9] J. M. Mendel : Optimal Seismic Deconvolution. An Estimation based approach, Academic Press; 1983

[10] J.M.Mendel and J.Kormylo: New fast optimal white-noise estimators for deconvolution, IEEE Trans. Geo. Elec., vol. GE-15, pp. 32-41, No. 1, January 1977.

[11] J.A. Jensen: A Model for the Propagation and Scattering of Ultrasound in Tissue, To be published in Journal of the Acoustical Society of America.

[12] G.E. Tupholme: Generation of acoustic pulses by baffled plane pistons, Mathematika 16, pp. 209-224, 1969.

[13] P.R. Stepanishen: The time-dependent force and radiation impedance on a piston in a rigid infinite planar baffle, J.Acoust.Soc.Am. 49 (3), pp. 841-849, 1971A.

[14] P.R. Stepanishen: Transient radiation from pistons in a infinite planar baffle, J.Acoust.Soc.Am. 49, pp. 1627-1638, 1971B.

[15] L. Ljung : System Identification. Theory for the user, Prentice-Hall Inc., 1987

[16] L. Ljung and T. Söderström : Theory and practice of recursive identification, The MIT press, 1983. 